\section{Fighting tumors with tumors}

\section{By Tracey Baas, Senior Editor}

Researchers at The Rogosin Institute have used cancer cell-containing agarose beads to inhibit tumor proliferation in animals. ${ }^{1}$ Although the strategy sounds counterintuitive, the macrobeads already are in an investigator-sponsored Phase II trial in patients with pancreatic cancer, advanced colorectal cancer or refractory prostate cancer.

Previous work had shown that the growth of solid tumors is characterized by progressive slowing of the rate of growth as the tumor increases in size, suggesting that growth might be inhibited by molecules signaling the presence of tumor mass.,3

Rogosin's Barry Smith and his team hypothesized that this negative feedback could be harnessed to fight cancer if the same effect could be recreated through controlled exposure to a tumor-for example, to tumor cells encapsulated in an artificial matrix.

Smith is director of Rogosin, a professor of clinical surgery at Weill Cornell Medical College and an attending physician at New York Presbyterian Hospital.

The researchers set out to test this hypothesis by growing mouse renal adenocarcinoma (RENCA) cells in agarose and then coating them with agarose to produce macrobeads that were $6-8 \mathrm{~mm}$ in diameter.

Within one week after encapsulation, $99 \%$ of the tumor cells underwent apoptosis. The remaining tumor cells represented several subpopulations.

These surviving cells formed large, metabolically active tumor colonies that could be maintained in the 3D culture indefinitely, with little increase in total cell number or colony volume but with continuous individual cellular turnover. Although tumor cells were proliferating, they exhibited automatic growth inhibition as the encapsulated tumor reached its ultimate mass in the macrobead.

Further studies showed that the cells displayed stem cell markers, had increased gene expression in pathways associated with stem cells and, when released from the 3D matrix after three years of encapsulation, could still proliferate and form tumors.

To discover what signals the encapsulated tumor cells were using for growth regulation, the team used mass spectroscopy to identify around 750 proteins and peptides in macrobead culture media. At least 10 of these proteins were highly conserved across species and known to have tumor inhibitory properties.

Thus, the researchers postulated that, in vivo, the inhibitory proteins could be released by the beads into the abdominal fluid and then the bloodstream, where they would be transported to a tumor and possibly regulate its growth.

They put their hypothesis to the test in mice that had 2,500 RENCA cells implanted under the renal capsule, which surrounds the kidney. Using this mouse model of renal cancer, intraperitoneal implantation of the macrobeads led to smaller tumors than implantation of empty macrobeads $(p<0.05)$.

The researchers also tested the macrobeads in dogs and cats in the end stages of various naturally occurring cancers. The beads improved disease in 39 of 51 animals without evident impairment of their immune systems. One cat with gastrointestinal lymphoma lived for three years after receiving five macrobead implants, and a cat with mammary carcinoma lived for eight years after receiving four implants. Each received about 200 beads at each implant site.

In 11 dogs with prostate cancer, the macrobeads increased median survival to 177 days compared with an expected survival of $<50$ days for no treatment.

Based on these data, Smith's team believes the macrobead effect to be neither species- nor tumor-specific.

The team published its findings in Cancer Research and included scientists from Cornell University, Columbia University, New York-Presbyterian Hospital, The Rockefeller University, The Ohio State University, Bob Evans Farms Inc., the Veterinary Oncology \& Hematology Center and the Gerald P. Murphy Cancer Foundation.

Richmond Prehn, professor emeritus of pathology at the University of Washington and one of the original proponents of the idea that tumor growth could be inhibited by tumor mass, ${ }^{2}$ thought the effect of multiple inhibition components from the macrobead to slow tumor growth was "exciting indeed."

He added that it was "very surprising that the presumably relatively small number of encapsulated tumor cells had such profound effects on the much larger tumor target in the in vivo experiments."

Indeed, he said those data could imply that the "confined cells were putting out unexpectedly large amounts of inhibition components."

"There are actually significant changes of up to 100 -fold in the amounts of particular proteins and/or peptides that are released by the cells in the macrobeads," Smith said. "In addition to the possibility of larger amounts of particular molecules being released, there is also the possibility that one or more of the released factors are triggering a cascade of events that help to produce part of the effect."

\section{The maturation of a macrobead}

Olga Garkavenko, head of molecular diagnostics at Living Cell Technologies Ltd., said the findings illustrate "the unique aspect of cellular therapy-the synergistic effect of multiple components as opposed to the action of just a single agent."

She said the technology described in the paper might be a good tool for tumor regulation research and for in vitro testing of proposed antitumor therapeutics because the cell population of the macrobeads seems to represent a cancer stem cell niche of sorts. Because cancer 
stem cells are believed to be responsible for enabling the perpetuation of tumors, the macrobeads could be used to test therapeutics that, to be optimally effective, would have to show activity against such cells.

Garkavenko did say that using the macrobeads as a therapeutic "might be a bit premature. It would be very useful to show the data on longterm tracking of tumor cells from macrobeads in experiments" to ensure that the cancerous xenocells do not survive in the host if they somehow escape from the macrobeads.

Previous work has shown that porcine cells can survive for years in a nonimmunosupressed patient after extracorporeal pig spleen perfusion. ${ }^{4}$

Smith acknowledged that there is a very small possibility of microchimerism-the survival of a xenocell in the recipient's bloodstream. However, he told SciBX that "the mechanical barriers of the agarose shell, immunologic-xenogeneic_-barriers of the host and macrobead growth-controlling signals in bloodstream all work to prevent cross-species seeding and propagation of unencapsulated cells. In fact, in our veterinary study of cats and dogs, no evidence of any surviving mouse cancer cells outside the macrobead is found for periods of up to 3-5 years post-implant."

In a Phase I trial in cancer patients, intraperitoneal implants of about 8-16 macrobeads per kilogram per patient were safe. Because the agarose beads are well tolerated by the body, there is no need to remove them. The human abdomen could hold as many as 15 implants without a problem.

The ongoing Phase II trial is enrolling patients with pancreatic cancer, advanced colorectal cancer or refractory prostate cancer and is measuring tumor response rate, time to progression, quality of life and tumor markers.

\section{The macrobead core}

The Rogosin team is not the only one using macrobeads in cancer. An endothelial cell-containing macrobead was described at the beginning of the year in Science Translational Medicine by researchers from the Massachusetts Institute of Technology, The University of Texas at Austin, the Boston University School of Medicine and Harvard Medical School. ${ }^{5}$

These researchers, led by Elazer Edelman, showed that endothelial cells encapsulated in gelatin matrices decreased tumor growth and invasiveness in mice. Pervasis Therapeutics Inc. is developing the implant technology to treat solid tumors in conjunction with surgical resection.

Edelman is a professor of health sciences and technology at MIT, professor of medicine at Harvard Medical School and a coronary care unit cardiologist at Brigham and Women's Hospital. He is also a cofounder and director of Pervasis and member of the scientific advisory board.

Although the technology might appear to be potentially safer because it uses endothelial cells instead of cancer cells, researchers interviewed by SciBX wanted to see next steps showing whether the cross talk occurring between the encapsulated endothelial cells and the host tumor cells could cause the transformation of the endothelial cells. ${ }^{6}$

Smith said, "There seem to be similarities between the two macrobead systems with multiple factors being secreted that act on multiple pathways to inhibit growth. Inhibiting a single pathway does not appear to be enough to inhibit cancer cell growth."

"It is possible," he continued, "that endothelial cells are having some effect in our model given that the RENCA cells are passaged through mice and such vasculature cells could be encapsulated in the RENCA macrobeads. However, I don't see that Edelman's system selects for stem cells."

Edelman believes that the idea of using cells as paracrine regulators is gaining momentum. "Cell-based therapies offer more physiologic control, far less toxicity than pharmacologic approaches and when embedded can take the form of therapeutic units that can be stored, used immediately and assayed for function prior to implantation," he said.

He added that "what is most exciting is that we and others have used these approaches effectively with mature, progenitor and stem cells."

Rogosin has exclusively licensed its macrobead IP to the Metromedia Bio-Science LLC unit of Metromedia Co.

Metromedia Co., the privately held broadcast and telecommunications company run by billionaire John Kluge until his death in September, is financially backing the studies.

Metromedia Bio-Science has put $\$ 50$ million into the cancer project and intends to funnel the bulk of any revenue from the treatment, should it reach the market, into Kluge's charitable foundation.

Baas, T. SciBX 4(7); doi:10.1038/scibx.2011.181

Published online Feb. 17, 2011

\section{REFERENCES}

1. Smith, B.H. et al. Cancer Res.; published online Jan. 24, 2011; doi:10.1158/0008-5472.CAN-10-2258

Contact: Barry H. Smith, The Rogosin Institute, New York, N.Y. e-mail: bas2005@nyp.org

2. Prehn, R.T. Cancer Res. 51, 2-4 (1991)

3. DeWys, W.D. Cancer Res. 32, 374-379 (1972)

4. Paradis, K. et al. Science 285, 1236-1241 (1999)

5. Franses, J.W. et al. Sci. Transl. Med. 3, 66ra5 (2011)

6. Haas, M.J. SciBX 4(5); doi:10.1038/scibx.2011.124

COMPANIES AND INSTITUTIONS MENTIONED

Bob Evans Farms Inc., Columbus, Ohio

Boston University School of Medicine, Boston, Mass.

Brigham and Women's Hospital, Boston, Mass.

Columbia University, New York, N.Y.

Cornell University, Ithaca, N.Y.

Gerald P. Murphy Cancer Foundation, West Lafayette, Ind.

Harvard Medical School, Boston, Mass.

Living Cell Technologies Ltd. (ASX:LCT; OTCQX:LVCLY),

Sydney, Australia

Massachusetts Institute of Technology, Cambridge, Mass.

Metromedia Bio-Science LLC, New York, N.Y.

New York-Presbyterian Hospital, New York, N.Y.

The Ohio State University, Columbus, Ohio

Pervasis Therapeutics Inc., Cambridge, Mass.

The Rockefeller University, New York, N.Y.

The Rogosin Institute, New York, N.Y.

The University of Texas at Austin, Austin, Texas

University of Washington, Seattle, Wash.

Veterinary Oncology \& Hematology Center, Norwalk, Conn.

Weill Cornell Medical College, New York, N.Y. 